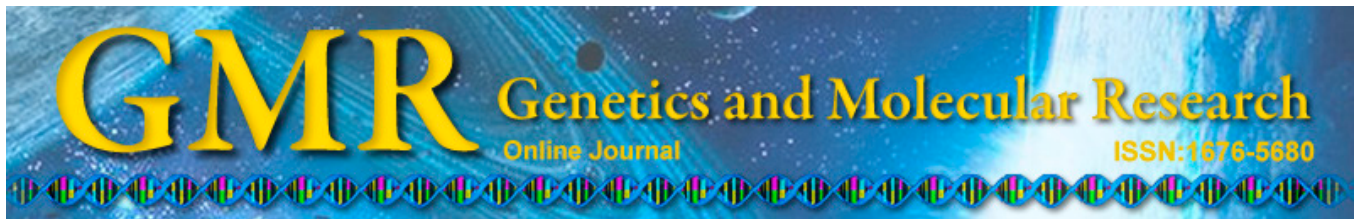

\title{
Electroacupuncture decreases the urinary bladder pressure in patients with acute gastrointestinal injury
}

\author{
H.J. Yu, J.G. Zhu, P. Shen, L.H. Shi, Y.C. Shi and F. Chen \\ Department of Intensive Care Unit, the First Hospital of Jiaxing, Jiaxing, China \\ Corresponding author: F. Chen \\ E-mail: cfly149@sohu.com
}

Genet. Mol. Res. 14 (1): 34-39 (2015)

Received November 22, 2013

Accepted September 20, 2014

Published January 15, 2015

DOI http://dx.doi.org/10.4238/2015.January.15.5

\begin{abstract}
The present study investigates the effects of electroacupuncture (EA) on urinary bladder pressure (UBP) in patients with acute gastrointestinal injury (AGI). Twenty patients with AGI admitted to the First Hospital of Jiaxing intensive care unit from December 2011 to June 2013 were evaluated. Conventional group patients $(\mathrm{n}=10)$ were administered moderate enteral nutritional support, and electroacupuncture group patients $(\mathrm{n}=10)$ were administered enteral nutritional support followed by EA at bilateral Zusanli (ST-36), Shangjuxu (ST-37), Hegu (LI4), and QuChi (LI-11) acupoints. UBP was then measured every 6 $\mathrm{h}$ and the serum creatinine once daily for 7 days. There were no statistically significant patient demographic differences in the study groups $(\mathrm{P}>0.05)$. The initial UBP of both patient groups was $\geq 12$ $\mathrm{mmHg}$. On days 6 and 7, the UBP significantly decreased in the EA group compared to the conventional group $(\mathrm{P}<0.05)$. The serum creatinine concentration on day 7 was significantly lower in the EA group than in the conventional group $(\mathrm{P}<0.05)$. Based on these
\end{abstract}


results, electroacupuncture contributed to gastrointestinal motility recovery in patients with AGI. This procedure may reduce UBP and provide organ-protective effects in AGI patients.

Key words: Electroacupuncture; Acute gastrointestinal injury; Urinary bladder pressure

\section{INTRODUCTION}

Acute gastrointestinal injury (AGI) is frequently reported in the intensive care unit (ICU), affecting up to $62 \%$ of critically ill patients (Mutlu et al., 2001; Reintam et al., 2009). Gastrointestinal function is severely affected in the condition; AGI is frequently associated with acute pancreatitis and infectious peritonitis (Reintam Blaser et al., 2012). Evidence increasingly indicates that AGI is closely associated with a poor clinical outcome in critically ill patients (Lam et al., 2007; Reintam et al., 2008).

The steady-state abdominal cavity pressure, known as intra-abdominal pressure (IAP), ranges $0-5 \mathrm{mmHg}$ and inversely varies with the intrathoracic pressure during normal respiration (Lee, 2012). Temporary IAP changes can be induced by numerous factors, including coughing, sneezing, and even loud singing. However, chronically increased IAP reportedly predisposes patients to various systemic disorders, and critically ill patients with persistent IAP elevation $12 \mathrm{mmHg}$ or greater are at particular risk (Cheatham et al., 2007). To date, the ability of transurethral bladder catheter pressure to accurately reflect IAP is well defined (Yol et al., 1998). The procedure is also noninvasive, and thus, is commonly used clinically to measure IAP.

Electroacupuncture (EA), which electrically stimulates acupoints derived from traditional Chinese medicine theory, is commonly used in clinical practice, particularly in mainland China, but there are no clinical trials evaluating the procedure at present. The current study evaluates the potential efficacy of EA in alleviating elevated IAP in critically ill patients diagnosed with acute gastrointestinal injury.

\section{MATERIAL AND METHODS}

\section{Patient information}

In total, 20 patients diagnosed with AGI and admitted to the First Hospital of Jiaxing ICU from December 2011 to June 2013 were included in this study. The inclusion criteria were as follows: aged $\geq 18$ years; AGI secondary to acute pancreatitis and/or gastrointestinal perforation surgical repair; clinically confirmed abdominal distension; poor tolerance to enteral nutrition, typified by vomiting or gastric retention $\geq 200 \mathrm{~mL}$ at least once; and ICU hospitalization greater than 1 week. The exclusion criteria included: administration of other drugs without the investigator's permission; persistent hypotension $\leq 90 \mathrm{mmHg}$ refractory to vasoactive agents; infectious dermatitis preventing administration of electroacupuncture; and a permanent cardiac pacemaker. Patients reporting severe adverse effects were also excluded. All patients provided written informed consent. This study was approved by the Ethics Committee of the First Hospital of Jiaxing. 


\section{Treatment}

Patients were randomly assigned two study groups using a random number table, a conventional group $(n=10)$ and electroacupuncture group $(n=10)$. Investigators played no role in patient allocation. Patients in both groups received moderate enteral nutritional support and gastrointestinal promotility agents, such as enteroclytic rhubarb (20-30 g per day). Electroacupuncture was performed on the electroacupuncture group at the bilateral Zusanli (ST-36), Shangjuxu (ST-37), Hegu (LI-4), and QuChi (LI-11) acupoints using an electronic acupuncture instrument (Huato SDZIV, Suzhou Medical Appliance Factory, Suzhou, China) at a $5.0 \mathrm{~Hz}$ continuous wave and $2-5 \mathrm{~V}$ for 30 min once daily for 7 days.

\section{Urinary bladder pressure measurement}

Urinary bladder pressure (UBP) was measured with a bladder pressure monitoring device (ABV300, Wolfe Tory Medical Inco., Salt Lake City, UT, China). Sodium chloride solution $(0.9 \%) 25 \mathrm{~mL}$ was administered into the urinary bladder through a catheter, and pressure was measured every $6 \mathrm{~h}$ for 7 days with a sensor located at midaxilla.

\section{Serum creatinine measurement}

Serum creatinine concentration was measured as previously described once daily for 7 days (Peake and Whiting, 2006).

\section{Statistical analysis}

Data was analyzed with the SPSS 13.0 software and reported as means \pm standard deviation. Student $t$-test compared inter-group data at a statistical significance of $\mathrm{P}<0.05$.

\section{RESULTS}

\section{Patient characteristics}

Table 1 summarizes the patient demographics, including gender, age, APACHEII score, associated etiology, noradrenalin administration, and the bladder volume. No statistical demographic differences were observed in the experimental groups $(\mathrm{P}>0.05)$.

\begin{tabular}{lcc} 
Table 1. Patient characteristics & & EA group \\
\hline & Conventional group & 8 \\
\hline Gender & & 2 \\
Male (N) & 7 & $58.7 \pm 18.4$ \\
Female (N) & $61.3 \pm 19.1$ & $24.9 \pm 7.5$ \\
Age (yrs) & $23.8 \pm 7.6$ & 5 \\
APACHE II score & 5 & 4 \\
Inducing factors & 5 & 1 \\
Acute pancreatitis (N) & $4.89 \pm 4.54$ & $4.60 \pm 3.52$ \\
Surgery of intestinal perforation (N) & $3044.44 \pm 2050.68$ & $2920.00 \pm 2023.09$ \\
$\quad$ Surgery of gastric perforation (N) & & \\
Noradrenalin (mg) & & \\
Input volume - output volume (mL) & & \\
\hline
\end{tabular}

$\mathrm{EA}=$ electroacupuncture. 


\section{Comparison of daily UBP}

The UBP of both experimental groups was normal ( $\geq 12 \mathrm{mmHg}$ ) prior to therapy. The UBP significantly decreased in the EA group compared to the conventional group at days 6 and $7(\mathrm{P}<0.05$, Table 2$)$.

\section{Table 2. Urinary bladder pressure in experimental groups.}

\begin{tabular}{lcccccccc}
\hline \multicolumn{7}{c}{ Urinary bladder pressure $(\mathrm{mmHg})$} \\
\hline Group & Baseline $(\mathrm{mmHg})$ & $\mathrm{D} 1(\mathrm{mmHg})$ & $\mathrm{D} 2(\mathrm{mmHg})$ & $\mathrm{D} 3(\mathrm{mmHg})$ & $\mathrm{D} 4(\mathrm{mmHg})$ & $\mathrm{D} 5(\mathrm{mmHg})$ & $\mathrm{D} 6(\mathrm{mmHg})$ & $\mathrm{D} 7(\mathrm{mmHg})$ \\
\hline Conventional group $^{\mathrm{a}}$ & $18.2 \pm 2.80$ & $18.1 \pm 2.77$ & $17.8 \pm 2.94$ & $16.6 \pm 3.57$ & $15.8 \pm 4.08$ & $15.4 \pm 3.31$ & $14.9 \pm 3.73$ & $14.1 \pm 3.67$ \\
EA $^{\mathrm{c}}$ group & $18.7 \pm 3.28$ & $18.5 \pm 3.24$ & $17.6 \pm 2.80$ & $15.8 \pm 3.05$ & $14.6 \pm 3.66$ & $13.2 \pm 3.08$ & $11.7 \pm 2.58 *$ & $10.9 \pm 2.28^{\mathrm{b}}$ \\
$t$ value & 0.265 & 0.297 & -0.156 & -0.539 & -0.693 & -1.539 & -2.232 & -2.344 \\
P value & 0.792 & 0.770 & 0.878 & 0.596 & 0.497 & 0.141 & 0.039 & 0.031 \\
\hline
\end{tabular}

${ }^{a}$ Conventional group was administered moderate enteral nutrition support. ${ }^{b} \mathrm{EA}$ group was administered moderate enteral nutrition support followed by electroacupuncture once daily at bilateral ST-36, ST-37, LI-4, and LI-11 acupoints. ${ }^{\mathrm{E}} \mathrm{EA}=$ electroacupuncture. $^{*} \mathrm{P}<0.05$, compared to the conventional group.

\section{Serum creatinine concentration}

Table 3 summarizes the daily serum creatinine concentrations. Compared to the conventional group, the serum creatinine was remarkably decreased in the EA group on day $7(\mathrm{P}<0.05)$.

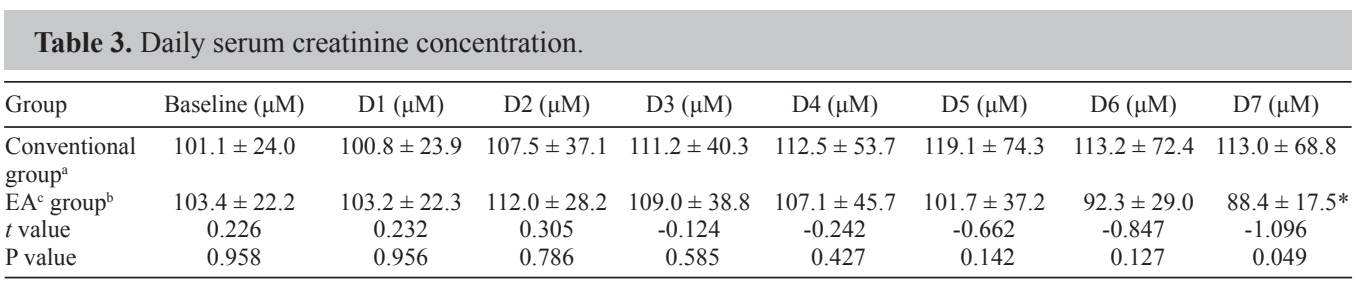

${ }^{a}$ Conventional group was administered moderate enteral nutrition support. ${ }^{b} E A$ group was administered moderate enteral nutrition support followed by electroacupuncture once daily at bilateral ST-36, ST-37, LI-4, and LI-11 acupoints. ${ }^{c} \mathrm{EA}=$ electroacupuncture. $* \mathrm{P}<0.05$, compared with conventional group.

\section{DISCUSSION}

AGI is commonly reported in critically ill patients and the causes associated are numerous. Among the most frequently causes reported of AGI is intestinal hypoperfusion, ischemia, and anoxia, which activates xanthine oxidase, increases oxygen radical accumulation, injures the intestinal mucosa, reduces digestive enzymatic activity, and decreases gastrointestinal digestion and absorption. Another common cause is endotoxin and inflammatory cytokine release, which decrease intestinal absorption and oxygen utilization, ultimately impairing intestinal mucosal recovery. Decreased enteric nervous system activity and gastroenteric dyskinesis are also cited, as is intestinal bacteria proliferation, which causes an accumulation of bacteria in the intestinal wall, generates metabolites and toxins, and injures the intestinal mucosa. Finally, activation of intestinal antigen presenting cells, platelet activating factor and tumor necrosis factor release, and decreased mucosal barrier protection also contribute to AGI.

The clinical symptoms of AGI are primarily abdominal distension, gastric retention, poor tolerance to enteral nutrition, and death (Mutlu et al., 2001; Reintam et al., 2009); IAP is 
considered an important index for gauging AGI severity. Generally, the normal IAP in humans is $5-7 \mathrm{mmHg}$, and a sharp IAP increase is associated with numerous conditions (De Keulenaer et al., 2009). For example, intra-abdominal hypertension (IAH) has been associated with an IAP $\geq 12 \mathrm{mmHg}$, while a higher risk of abdominal compartment syndrome (ACS) was reported in patients with an IAP $\geq 20 \mathrm{mmHg}$ (Vidal et al., 2008). In a multicenter epidemiological study performed in 6 countries analyzing the IAH prevalence in the intensive care unit, Malbrain et al. (2004) reported that 57 critically ill patients (58.8\%) were diagnosed with IAH and 8 $(8.2 \%)$ with ACS. In a previous study, an IAP increase of up to $20 \mathrm{mmHg}$ decreased the mesenteric perfusion by $40 \%$, and an increase to $40 \mathrm{mmHg}$ decreased the mesenteric perfusion by $70 \%$; intestinal permeability portal vein endotoxin concentration both remarkably increased in response (Lee, 2012). A persistent IAP $\geq 15 \mathrm{mmHg}$ is a reported independent risk factor for renal impairment. Acute IAP increase has been associated with respiratory, cardiovascular, and central nervous system disorders. Notably, IAP induced intra-abdominal hypertension has a well-known correlation to elevated mortality in critically ill patients.

Compared to manual acupuncture, electroacupuncture has several advantages such increased control and easier standardization, enabling stronger and continuous stimulation with less pain and tissue injury (Yeung et al., 2009). Electroacupuncture purportedly improves gastrointestinal function by enhancing gastrointestinal electric activity, increasing mucosa perfusion, and restoring immune and endocrine regulation. Under suitable electroacupuncture stimulation, functional disorders and metabolic disturbances may improve. In rats with reduced gastric mucosal blood flow (GMBF) caused by gastric mucosal injury, electroacupuncture performed at the Sibai (ST-2), Tianshu (ST-25), and Zusanli (ST-36) acupoints, GMBF increased after Sibai (ST-2) and Zusanli (ST-36) EA (Lin et al., 2003). A similar study evaluating EA in rabbits showed increased motilin and cholecystokinin concentrations in the smooth muscle, sphincter, and gastric antrum. In a randomized controlled trial investigating EA effects on critically ill patients with delayed gastric emptying (Chang et al., 2006), the gastric residual volume decreased from $970 \pm 87 \mathrm{~mL}$ to $346 \pm 71 \mathrm{~mL}(\mathrm{P}=0.003)$ after EA stimulation (Pfab et al., 2011).

In our study, the UBP was greater than $12 \mathrm{mmHg}$ in both groups, with no statistical difference $(\mathrm{P}>0.05)$. After EA treatment, the UBP significantly decreased in the EA group compared to the conventional group, specifically on days 6 and 7. In addition, on day 7, the serum creatinine in the EA group was significantly decreased compared to the conventional group. Collectively, these results demonstrate that EA contributed to gastrointestinal motility recovery in the critically ill patients. Electroacupuncture may also decrease the IAP, which may have protective effects for the organs.

\section{Conflicts of interest}

The authors declare no conflict of interest.

\section{ACKNOWLEDGMENT}

Research supported by the Bureau of Science and Technology, Jiaxing, Zhejiang (\#2011AY1050-9) and the Traditional Chinese Medicine Scientific Research Fund from Zhejiang (\#2011ZA103). 


\section{REFERENCES}

Chang X, Jie Y, Liu Y, Zhang H, et al. (2006). Effects of electroacupuncture at Zuyangmin and Zushaoyang Jingxue on stomach and gallbladder kinases and related brain-gut peptide in rabbits. Worl Chin J. Dige 14: 1662-1668.

Cheatham ML, Malbrain ML, Kirkpatrick A, Sugrue M, et al. (2007). Results from the International Conference of Experts on Intra-abdominal Hypertension and Abdominal Compartment Syndrome II Recommendations. Intensive Care Med. 33: 951-62.

De Keulenaer BL, De Waele JJ, Powell B and Malbrain ML (2009). What is normal intra-abdominal pressure and how is it affected by positioning, body mass and positive end-expiratory pressure? Intensive Care Med. 35: 969-76.

Lam SW, Nguyen NQ, Ching K, Chapman M, et al. (2007). Gastric feed intolerance is not increased in critically ill patients with type II diabetes mellitus. Intensive Care Med. 33: 1740-5.

Lee RK (2012). Intra-abdominal hypertension and abdominal compartment syndrome: a comprehensive overview. Crit Care Nurse 32: 19-31.

Lin Y, Yi S and Yan G (2003). Effect of stimulating points of the stomach meridian of foot-yangming by electronic needle on gastric mucosal blood flow and somatostatin in the rat model of gastric mucosal injury. Chin. J. Inte. Med. 11: 75-77.

Malbrain ML, Chiumello D, Pelosi P, Wilmer A, et al. (2004). Prevalence of intra-abdominal hypertension in critically ill patients: a multicentre epidemiological study. Intensive Care Med. 30: 822-9.

Mutlu GM, Mutlu EA and Factor P (2001). GI complications in patients receiving mechanical ventilation. Chest 119: $1222-41$.

Peake M and Whiting M (2006). Measurement of serum creatinine-current status and future goals. Clin. Biochem Rev 27: 173-84.

Pfab F, Winhard M, Nowak-Machen M, Napadow V, et al. (2011). Acupuncture in critically ill patients improves delayed gastric emptying: a randomized controlled trial. Anesth. Analg. 112: 150-5.

Reintam A, Parm P, Kitus R, Starkopf J, et al. (2008). Gastrointestinal failure score in critically ill patients: a prospective observational study. Crit. Care 12: R90.

Reintam A, Parm P, Kitus R, Kern H, et al. (2009). Gastrointestinal symptoms in intensive care patients. Acta Anaesthesiol. Scand. 53: 318-24.

Reintam Blaser A, Malbrain ML, Starkopf J, Fruhwald S, et al. (2012). Gastrointestinal function in intensive care patients: terminology, definitions and management. Recommendations of the ESICM Working Group on Abdominal Problems. Intensive Care Med. 38: 384-94.

Vidal MG, Ruiz Weisser J, Gonzalez F, Toro MA, et al. (2008). Incidence and clinical effects of intra-abdominal hypertension in critically ill patients. Crit Care Med. 36: 1823-31.

Yeung WF, Chung KF, Zhang SP, Yap TG, et al. (2009). Electroacupuncture for primary insomnia: a randomized controlled trial. Sleep 32: 1039-47.

Yol S, Kartal A, Tavli S and Tatkan Y (1998). Is urinary bladder pressure a sensitive indicator of intra-abdominal pressure? Endoscopy 30: 778-80. 